\title{
BMJ Open Treatment of febrile geriatric patients with suspected urinary tract infections in a hospital with high rates of ESBL producing bacteria: a cohort study
}

\author{
Zvi Shimoni, ${ }^{1,2}$ Regev Cohen, ${ }^{3}$ Ruslan Avdiaev, ${ }^{1}$ Paul Froom ${ }^{4,5}$
}

To cite: Shimoni Z, Cohen R, Avdiaev R, et al. Treatment of febrile geriatric patients with suspected urinary tract infections in a hospital with high rates of ESBL producing bacteria: a cohort study. BMJ Open 2016;6:e013696. doi:10.1136/bmjopen-2016013696

- Prepublication history for this paper is available online. To view these files please visit the journal online (http://dx.doi.org/10.1136/ bmjopen-2016-013696).

Received 2 August 2016 Revised 12 September 2016 Accepted 8 November 2016

CrossMark

For numbered affiliations see end of article.

Correspondence to Professor Paul Froom; froomp@gmail.com

\section{ABSTRACT}

Purpose: To determine the consequences of treating febrile geriatric patients with a suspected urinary tract infection (UTI) with antibiotics that have high resistance rates due primarily to extended-spectrum $\beta$-lactamase (ESBL) producing bacteria.

Methods: In this cohort study, we selected 257 consecutive hospitalised patients aged $\geq 70$ years with a chief symptom of fever, possibly due to a UTI and initially treated with antibiotics with rates in our hospital of urinary culture resistance $>20 \%$. Patients with severe sepsis were excluded. The main outcomes measures were in vitro bacterial resistance to initial antibiotic therapy (BRIAT), response to therapy, hospitalisation days and mortality.

Results: Urine cultures were positive in $64.2 \%$ (165 of 257) of the patients and BRIAT occurred in $28.0 \%$ (72 of 257). Response rates were 100\% (93 of 93) in those with bacteria sensitive to initial antibiotic therapy, $95.7 \%$ (88 of 92 ) in the culture negative patients, and $66.7 \%$ (48 of 72$)$ in those with BRIAT $(p<0.001)$. There were no deaths due to deterioration during the initial treatment period because of BRIAT. In the patients with BRIAT, the median length of hospitalisation was 3 days longer than that in the other patients ( 7 and 4 days, respectively, $p<0.001$ ).

Conclusions: We conclude that initial broad spectrum antibiotic treatment could potentially lower the median length of hospitalisation by 3 days in many hospitalised geriatric patients without an extra-urinary tract source for their fever. This benefit needs to be balanced against the risk to the individual patient and to the general public of increasing bacterial resistance rates to broader spectrum antibiotics often held in reserve.

\section{INTRODUCTION}

Urinary tract infections (UTI) are a common reason for hospital admission in febrile geriatric patients, and prompt initiation of empiric antimicrobial therapy can improve clinical outcomes. ${ }^{1}$ Today, however, there are many European countries with extended-

\section{Strengths and limitations of this study}

- This cohort study included all geriatric febrile patients with a suspected urinary tract infection (UTI) rather than only those who were diagnosed with a UTI in retrospect.

- We focused on the proper treatment of patients with a febrile UTI, and therefore excluded those with severe urosepsis, who require initial treatment with broad spectrum antibiotics.

- It is unclear how referral and admission practices might have influenced the results.

- We did not have information on recent antibiotic therapy before hospitalisation that might have affected our ability to predict bacterial resistance.

- A much larger cohort is needed to rule out an early mortality risk from BRIAT.

spectrum $\beta$-lactamase (ESBL) producing bacteria rates of over $40 \% ;^{2}$ in such settings, it is unclear whether to accept a significant risk for bacterial resistance to initial antibiotic therapy (BRIAT) or to treat the patient with wider spectrum antibiotics that are generally held in reserve in order to decrease future resistance rates. ${ }^{3-6}$ Despite high rates of ESBL producing bacteria in our hospital, we reserve initial treatment with amikacin or a carbapenem (antibiotics with very low resistance rates in our hospital) for patients with either severe sepsis, ${ }^{7}$ or those who have a history of a bacterial resistant infection. Physicians most commonly prescribe either ceftriaxone or cefuroxime.

In order to study the consequences of our hospital policy, we selected geriatric patients hospitalised with a chief symptom of fever and a suspected UTI. Excluded were patients with findings on admission consistent with an infection outside the urinary tract, and those with an indication for empiric wide spectrum antibiotics (eg, severe sepsis). We determined the proportion of patients with BRIAT, the 72-hour response rate to 
antibiotics, the length of hospitalisation and associated early mortality.

\section{METHODS}

In this historical prospective study, we selected consecutive patients aged 70 years or older with a chief symptom of fever hospitalised in one of the three internal medicine departments from June 2012 to the end of August 2013. Excluded were patients with severe sepsis, ${ }^{7}$ or those with a source of fever on admission outside the urinary tract (those with new pulmonary infiltrates or an infiltrate in a patient without a previous chest X-ray (CXR), a cough with or without shortness of breath and no definite infiltrate on CXR, purulent bedsores, new areas of cellulitis and a presentation of vomiting and/or diarrhoea consistent with gastrointestinal tract infections or other conditions whose presentation suggested another diagnosis) (table 1). Finally, we excluded those with a history of a UTI due to resistant bacteria who received initial treatment with broad spectrum antibiotics with urine culture resistance rates $<10 \%$ (rates in our hospital over the past 18 months $-8.1 \%$ for piperacillin/ tazobactam, $1.9 \%$ for amikacin and $2.1 \%$ for ertapenem). All other antibiotics had resistance rates $>20 \%$. Also excluded were patients who did not receive antibiotics on admission.

Admission-independent variables included age, gender, being chronically bedridden for those who need help to get out of bed (nearly always nursing home patients), patients being treated for diabetes mellitus, any history of a treated UTI mentioned in the chart, urinary tract symptoms (dysuria, frequency or lower abdominal pain), the presence or absence of a permanent urinary catheter on admission, the need for a urinary catheter to obtain a urine specimen, the residual

Table 1 Included and excluded febrile geriatric patients by discharge diagnosis

\begin{tabular}{lc}
\hline Diagnosis & $\mathbf{N}(\%)$ \\
\hline Urinary tract infection & $192(27.0)^{\star}$ \\
Pneumonia & $114(16.0) \dagger$ \\
Lower respiratory tract infection & $111(15.7) \dagger$ \\
Culture-negative yet treated & $100(14.1) \dagger$ \\
Severe sepsis & $44(6.4) \dagger$ \\
Not treated-viral infection? & $39(5.6) \dagger$ \\
Cellulitis & $36(5.1) \dagger$ \\
Infected pressure sores & $27(3.7) \dagger$ \\
Gastrointestinal disease & $24(3.4) \dagger$ \\
Other§ & $21(3.0) \dagger$ \\
Total & 708 \\
\hline
\end{tabular}

*27 patients were excluded because they received broad spectrum antibiotics on admission.

†Excluded from the study.

‡Eight patients were excluded because they received broad spectrum antibiotics on admission.

$\S$ Endocarditis $(n=2)$, gall bladder infection $(n=5)$, collagen vascular disease $(n=9)$, cancer $(n=3)$, and fever $(n=2)$. urine volume in those catheterised, chronic dementia (recorded in the chart), active cancer or present treatment with steroids, and admission temperature to the internal medicine department.

Patients were divided into those with and without BRIAT. The diagnosis of a UTI required a history of fever, and a positive urine or blood culture ${ }^{8}$ but without a source of fever outside the urinary tract.

Significant bacteriuria was defined as the presence of $10^{5}$ colony forming units per millilitre of urine. External quality control (College of American Pathologists External Quality Assurance Proficiency Testing) did not include ESBL testing but all bacterial resistance $(n=28)$ and bacterial sensitivity $(n=64)$ to various antibiotics were identified correctly over the past 4 years. Internal quality controls identified correctly (AST-N270, BioMerieux) 36 positive and 36 negative ESBL test results performed over the past 3 years.

BRIAT was defined as initiation of treatment with an antimicrobial agent to which the infecting bacteria was ultimately shown not to be susceptible in vitro. The major outcome variable was the response to therapy that was defined by the absence of fever, or a trend for decreasing body temperatures by 72 hours when the results of urine cultures were available and the decision for continuing or changing antibiotic therapy was made. Other outcome variables were total hospitalisation days, prolonged hospitalisation (hospital days $\geq 7$ ) and in-hospital mortality. All deaths were reviewed by two of the authors (ZS and PF) independently and it was determined if mortality was related to BRIAT.

\section{Statistical analysis}

We calculated proportions, medians, quartiles, $95 \%$ CIs, means and SDs. The $\chi^{2}$ test was used to compare differences between proportions. For small numbers, Fisher's exact test was substituted. The Kruskal-Wallis test that does not assume a normal distribution was used for comparing continuous data. A $p$ value of $<0.05$ was considered statistically significant. We used logistic regression analysis to predict those with BRIAT and those with prolonged hospitalisations. All independent variables were entered into the model and retained only if they added significantly. Then non-significant variables were added back one at a time and again retained only if they added significantly to the model. Finally, the area under the curve was calculated. The model is considered fair if the area under the curve is $\geq 70 \%$, good if $80-89 \%$ and excellent if $90 \%$ or more.

\section{RESULTS}

There were 708 patients $(10.8 \%)$ who presented to the ED with a chief symptom of fever out of 6588 patients aged 70 years or older, hospitalised in the internal medicine departments. The mean age of the patients was 83 \pm 7 years in those with and without a chief symptom of fever, but there were fewer women in those with fever 
(50\% (352 of 708 compared to $56 \%$ (3294 of 5880), $\mathrm{p}<0.001)$ ).

The study group included 257 patients without a source for their fever outside the urinary tract and who were treated empirically with antibiotics; $165(64.2 \%)$ were culture positive and $72(28.0 \%)$ had BRIAT according to the culture results $(43.6 \%(72 / 165)$ of those with positive culture results). Since in vitro urinary culture resistance rates to either amikacin or ertapenem in our hospital are around 2\%, the estimated decreased rate of BRIAT is $26 \%(28 \%-2 \%)$ if the patients were treated empirically with one of those antibiotics.

Resistance was due to ESBL positivity in 62 patients and there were another 10 ESBL-negative patients with resistance to treatment with ceftriaxone $(n=9)$, and amoxicillin/clavulanate $(\mathrm{n}=1)$. The resistant bacteria included Proteus spp $(\mathrm{n}=2)$, Klebsiella pneumoniae $(\mathrm{n}=2)$, Pseudomonas aeruginosa $(\mathrm{n}=2)$ and Enterococci $\operatorname{spp}(\mathrm{n}=4)$.

Those with BRIAT had significantly more patients who were bedridden, demented and had a permanent urinary catheter or a history of a UTI (table 2). A logistic regression model showed that being bedridden and having a permanent urinary catheter increased the odds of BRIAT (OR 3.00 (95\% CI 1.52 to 5.90) and OR 2.39 (95\% CI 1.26 to 4.55$)$ ), respectively. However, the logistic regression model was poorly predictive of BRIAT with an AUC of only $68.3 \%$ (95\% CI- $67.5 \%$ to $69.1 \%$ ). There were 95 patients who were not bedridden and

Table 2 Patients with no source for fever outside the urinary tract

\begin{tabular}{|c|c|c|c|}
\hline Variables & $\begin{array}{l}\text { Others, } \\
\text { N=185, } \\
\text { n (\%) }\end{array}$ & $\begin{array}{l}\text { BRIAT, } \\
\text { N=72, } \\
\text { n (\%) }\end{array}$ & p Value \\
\hline Age (years) & $83 \pm 7$ & $85 \pm 5$ & $<0.005$ \\
\hline Female & $94(50.8)$ & $42(58.3)$ & 0.278 \\
\hline Bedridden & $97(52.4)$ & $58(80.6)$ & $<0.001$ \\
\hline Demented & $100(54.1)$ & 47 (65.3) & 0.103 \\
\hline $\begin{array}{l}\text { Permanent urinary } \\
\text { catheter }\end{array}$ & $30(16.2)$ & $28(38.9)$ & $<0.001 \dagger$ \\
\hline $\begin{array}{l}\text { Provided a urine } \\
\text { specimen }\end{array}$ & $52(28.1)$ & $9(12.5)$ & 0.008 \\
\hline $\begin{array}{l}\text { Catheterised for } \\
\text { specimen }\end{array}$ & $103(55.7)$ & $35(48.6)$ & 0.331 \\
\hline $\begin{array}{l}\text { Residual volume } \\
>300 \mathrm{~mL}\end{array}$ & $28(15.1)$ & $8(11.1)$ & 0.404 \\
\hline $\begin{array}{l}\text { Residual } \\
\text { volume } \geq 1000 \mathrm{~mL}\end{array}$ & $5(2.7)$ & $3(4.2)$ & $0.398 \ddagger$ \\
\hline Diabetes mellitus & $58(31.4)$ & $25(34.7)$ & 0.604 \\
\hline History of UTI & $101(54.6)$ & $56(77.8)$ & $<0.001$ \\
\hline $\begin{array}{l}\text { Urinary tract } \\
\text { symptoms }\end{array}$ & $49(26.5)$ & $8(11.1)$ & 0.008 \\
\hline Temperature $\geq 37.8^{\circ} \mathrm{C}$ & 154 (83.2) & $57(79.2)$ & 0.444 \\
\hline \multicolumn{4}{|c|}{$\begin{array}{l}\text { BRIAT, bacterial resistance to initial antibiotic therapy; UTI, urinary } \\
\text { tract infection. } \\
{ }^{*} p<0.001 \text { when considered overall for the three alternative ways of } \\
\text { providing a urine sample. } \\
\text { †Fisher's exact test. }\end{array}$} \\
\hline
\end{tabular}

without a permanent catheter; $11(11.6 \%)(95 \%$ CI $5.2 \%$ to $18.0 \%$ ) had BRIAT.

Initial therapy was primarily with ceftriaxone $(n=203)$ or cefuroxime $(n=23)$, but there were a few patients treated with ciprofloxacin $(n=8)$, ofloxacin $(n=6)$, gentamicin $(n=10)$, amoxicillin/clavulanate $(n=5)$ or ampicil$\operatorname{lin}(\mathrm{n}=2)$.

Escherichia coli infections were by far the most common cause of the culture-positive patients, and together with Klebsiella pneumoniae and Proteus spp made up over $90 \%$ of the bacterial species (table 3). There were 32 positive blood cultures, with the same species found in the urine in 24 cases. The other eight patients had negative or mixed urine cultures but positive blood cultures (E. coli 5, K. pneumoniae 2 and Proteus spp 1). ESBL positivity was found in $40.0 \%$ (66 of 165).

Response rates were $100 \%$ (93 of 93) in those with bacteria sensitive to initial antibiotic therapy, $95.7 \%$ (88 of 92) in the culture-negative patients, and $66.7 \%$ (48 of $72)$ in those with BRIAT (table 4$)(\mathrm{p}<0.001)$. Those with BRIAT had a median hospitalisation time of 7 days (1st3rd quartiles $=4-8$ days) compared to 4 days for the rest of the cohort (1st-3rd quartiles $=3-6$ days $) \quad(p<0.001)$, which was the same for culture-positive and culturenegative patients. The proportion of prolonged hospitalisations was also nearly identical in culture-positive patients without BRIAT and in culture-negative patients (table 4). The prolonged median hospitalisation time in those with BRIAT might in part have been due to more patients being bedridden and with a permanent catheter in the BRIAT group. However, a logistic regression model showed that BRIAT increased the odds for a hospitalisation time of 7 days or more by 5.23 -fold (95\% CI 2.75 to 9.91) after adjustment for being bedridden and for having a permanent catheter (variables that did not add significantly to the model).

The in-hospital death rate in those with a culture positive UTI was $1.2 \%$ ( 3 of 165) but BRIAT might have increased the risk in only one 89-year-old male patient who had a UTI with ESBL producing E. coli, responded to treatment with ceftriaxone, but on day 4, 1-day after treatment, was changed to ertapenem (according to

Table 3 Bacterial cultures in 165 patients with a culture-positive UTI

\begin{tabular}{lc}
\hline Bacteria & N (\%) \\
\hline E. coli & $113(68.5)$ \\
Klebsiella pneumoniae & $21(12.7)$ \\
Proteus spp & $18(10.9)$ \\
Pseudomonas aeruginosa & $5(3.0)$ \\
Enterococci spp & $5(3.0)$ \\
Other & $3(1.8)$ \\
ESBL positivity* & $66(40.0)$ \\
\hline
\end{tabular}

UTI, urinary tract infection; ESBL, extended-spectrum $\beta$-lactamase.

${ }^{\star} \mathrm{ESBL}$ producing bacteria. 
Table 4 Response rates of selected febrile geriatric patients without a source of fever outside the urinary tract and treated with antibiotics that have high urinary culture resistance rates

\begin{tabular}{lllll}
\hline $\begin{array}{l}\text { Final } \\
\text { diagnosis }\end{array}$ & $\mathbf{N}$ & $\begin{array}{l}\text { Response, } \\
\mathbf{n}(\%)\end{array}$ & $\begin{array}{l}\text { Hospital } \\
\text { days } \geq 7, \\
\mathbf{n}(\%)\end{array}$ & $\begin{array}{l}\text { Deaths, } \\
\mathbf{n}(\%)\end{array}$ \\
\hline $\begin{array}{l}\text { Culture } \\
\text { positive }\end{array}$ & 165 & $140(84.8)$ & $51(30.9)$ & $3(1.8)$ \\
BRIAT & 72 & $48(66.7)$ & $37(51.4)$ & $2(2.8)$ \\
Sensitive & 93 & $93(100)^{*}$ & $14(15.1)^{*}$ & $1(1.1)$ \\
$\begin{array}{l}\text { Culture } \\
\text { negative }\end{array}$ & 92 & $88(95.7)$ & $14(15.2)$ & $0(0.0)$ \\
\hline $\begin{array}{l}\text { BRIAT, bacterial resistance to initial antibiotic therapy. } \\
\text { *Compared to BRIAT, p<0.001. }\end{array}$
\end{tabular}

culture sensitivities) and died from septic shock. The other two patients responded to antibiotics; one died of aspiration pneumonia and the other from multiple complications 20 days later unrelated to the UTI. There were no deaths in the culture-negative patients.

\section{DISCUSSION}

The main finding of our study is that initial broad spectrum antibiotic treatment could potentially lower the median length of hospitalisation by 3 days in $26 \%$ of selected hospitalised geriatric patients without an extraurinary tract source for their fever. However, most patients with BRIAT responded to therapy, and no patient became septic before the availability of urinary culture results. To the best of our knowledge, this is the first cohort study of geriatric febrile patients that included all those with a suspected UTI.

Esparcia et $a l^{8}$ retrospectively studied geriatric patients whose primary diagnosis on discharge was a UTI; they reported an in-hospital mortality rate of $8.9 \%$ and found that 'inadequate empirical antimicrobial therapy' was an independent risk factor for mortality (OR 3.47, 95\% CI 1.42 to 8.48 ). They did not, however, separate out patients presenting with severe urosepsis or control for it in their models. Their findings support the need for wide spectrum antibiotics in those with severe urosepsis, ${ }^{9}$ but cannot be extrapolated to patients with uncomplicated febrile UTIs.

Our results support a recent meta-analysis estimation of no definite increase in the risk for mortality in patients of all ages treated with BRIAT on internal medicine wards for upper UTIs. ${ }^{10}$ Similarly, Peralta et al reported that antibiotic resistance for patients with bacteraemia due to ESBL producing E. coli or Klebsiella spp was not associated with mortality in patients without severe sepsis or septic shock. ${ }^{11}$

We found that two-thirds of the patients responded to treatment despite BRIAT, and those who did not respond were then given antibiotics according to sensitivities after the availability of the culture results. The death rate in those with positive cultures was $1.8 \%$ (3 of 165) but delayed therapy with broad spectrum antibiotics might have decreased the prognosis in one patient. This is consistent with two studies that reported response rates similar to ours in patients with BRIAT and no increase in short-term mortality, even though the antibiotics were often not changed after culture results became available. ${ }^{12} 13$ Whether or not antibiotics should be changed in responsive patients with BRIAT needs to be studied.

This study has a number of limitations. Our ability to predict patients with BRIAT was poor, although the odds were higher in those who were bedridden or had a permanent urinary catheter, consistent with previous reports attempting to predict the presence of ESBL. ${ }^{14}$ The rate of BRIAT decreased to $11.6 \%$ in patients not bedridden and without a permanent urinary catheter, but it is unclear how this might affect treatment decisions. We did not have the history of recent antibiotic usage that has been consistently shown to increase the risk for ESBL positivity. ${ }^{14}$

Extrapolation to other settings should be performed with caution because of possible selection bias. The general policy of the emergency department is to consider admitting all patients $\geq 65$ years old with fever, but it is unclear how actual referral and admission practices might have influenced the results. Our cohort also did not include patients with neutropenic fever or with haematological malignancies who were hospitalised in the Haematology department or patients with known lesions in the urinary tract who were hospitalised in surgery departments. Nevertheless, it is likely that our results can be extrapolated to other uncomplicated hospitalised geriatric patients whose chief symptom is fever. Our study was also limited by the fact that mortality was considered only during the hospitalisation period. Asakura et $a l^{12}$ followed up hospitalised patients with ESBL-positive UTIs for 4 weeks after discharge and found no significant differences in the 14-day mortality rate or the rate of clinical cures at 1 month in those with or without BRIAT, even though they did not change treatment after the culture results were available if the patient responded to therapy. Further studies with longer follow-up periods are warranted.

Finally, there is no gold standard for the diagnosis of an upper UTI. We used the chief symptom of fever as a selector for suspected upper UTIs that required intravenous antibiotics, and excluded all those with another possible source of infection. Fever in a patient with a UTI is commonly considered to differentiate between those with pyelonephritis from a bladder infection. ${ }^{13}$ Although the patients came to the hospital because of fever, not all our patients had fever on admission; the proportion of culture-positive patients was nearly identical to that in those with an elevated body temperature on admission (results not shown), but the reliability of the history of fever was not studied. In fact, we cannot rule out overdiagnosis and underdiagnosis of an upper 
UTI that is common in geriatric patients. $^{15} 16$ Overdiagnosis is due to the high prevalence of asymptomatic bacteriuria that affects $7-10 \%$ of men and $17-20 \%$ of women and even higher proportions of institutionalised non-catheterised older people. ${ }^{15}{ }^{16}$ Underdiagnosis might have resulted from exclusion of culture-positive patients with other sources of infection but where the source of the fever was actually the urinary tract. The diagnosis of the 92 patients who received treatment despite negative culture results, most without urinary tract symptoms, is also unclear. However, owing to the very high response rate, it is likely that many of these patients did not have a UTI and might have been treated unnecessarily. It is uncertain, however, how to select patients who do not require initial antibiotic therapy.

The strength of our historical prospective study is that we had a clearly defined cohort, not selected by outcome variables. We were thus able to define the potential benefits of a policy of using wide spectrum antibiotics on admission in these selected patients and found that treatment of all patients with amikacin or a carbapenem could potentially decrease the number of hospitalisation days by a median of 3 days in $26 \%$ of the patients; this is consistent with the estimate by Parienti et $a l^{10}$ who assumed 4 extra days of hospitalisation in internal medical department patients who received BRIAT.

Our bacterial sensitivity profiles have been stable over the past 18 months with very low resistance rates for amikacin and ertapenem (results not shown), but it is unclear whether this is due in part to our policy of limiting the use of those antibiotics. It has been shown that a strong risk factor for infection with carbapenem-resistant bacteria is previous use of a carbapenem, ${ }^{3}$ and even brief exposure to a carbapenem increases the risk of colonisation with imipenem-resistant Gram-negative bacteria in patients under intensive care. ${ }^{4}$

We conclude that initial treatment with antibiotics that have high in vitro resistance rates is reasonable in selected elderly patients with fever and without an extraurinary tract source on admission, although there is a risk for longer hospitalisation times. This decision, however, is dependent on the unknown estimated risk to the patient and to the general population of treating all febrile patients with a suspected UTI on admission with broader spectrum antibiotics.

Increasing bacterial resistance primarily from ESBL-producing Enterobacteriaceae has occurred in Israel, most of Europe, the USA, Asia and elsewhere. ${ }^{2} 1718$ The prevalence, however, is variable and resistance difficult to predict because a substantial portion of community-onset, ESBL-producing infections occur among patients without discernible healthcare-associated risk factors. ${ }^{19}$ There are, however, some geographical regions with very low rates of bacterial resistance ${ }^{20}$ thought to be due to restrictive antibiotic use, but even in those regions resistance rates are increasing. ${ }^{18}$ Our findings suggest that it is acceptable to hold in reserve broad spectrum antibiotics that cover ESBL producing infections in elderly patients with fever regardless of the patients' geographic region.

Author affiliations

${ }^{1}$ Department of Internal Medicine B, Laniado Hospital, Netanya, Israel

${ }^{2}$ Ruth and Bruce Rappaport School of Medicine, Haifa, Israel

${ }^{3}$ Infectious Disease Department, Laniado Hospital, Netanya, Israel

${ }^{4}$ Department of Clinical Utility, Laniado Hospital, Netanya, Israel

${ }^{5}$ School of Public Health, University of Tel Aviv, Tel Aviv, Israel

Acknowledgements The authors thank Professor Jochanan Benbassat, JDC Brookdale Institute, Health Policy Research Program, Jerusalem for his initial critical review of the manuscript.

Contributors All authors contributed significantly to this manuscript, including contributions in the study design (ZS, RC, RA, PF), chart reviews (ZS, RA, PF), interpretation of the data (ZS, RC, RA, PF), and preparation of the manuscript (ZS, RC, RA and PF).

Competing interests None declared.

Ethics approval We received approval from the hospital ethics committee21.1.2014-0003-14LND. The Israeli Ministry of Health reviews the decisions of the hospital's ethics committee.

Provenance and peer review Not commissioned; externally peer reviewed.

Data sharing statement no additional data are available.

Open Access This is an Open Access article distributed in accordance with the Creative Commons Attribution Non Commercial (CC BY-NC 4.0) license, which permits others to distribute, remix, adapt, build upon this work noncommercially, and license their derivative works on different terms, provided the original work is properly cited and the use is non-commercial. See: http:// creativecommons.org/licenses/by-nc/4.0/

\section{REFERENCES}

1. Norman DC. Fever in the elderly. Clin Infect Dis 2000;31:148-51.

2. Jones RN, Flonta M, Gurler N, et al. Resistance surveillance program report for selected European nations (2011). Diagn Microbiol Infect Dis 2014;78:429-36.

3. Chang HJ, Hsu PC, Yang CC, et al. Risk factors and outcomes of carbapenem-nonsusceptible Escherichia coli bacteremia: a matched case-control study. J Microbiol Immunol Infect 2011;44:125-30.

4. Armand-Lefevre L, Angebault C, Barbier F. Emergence of imipenem-resistant Gram-negative bacilli in intestinal flora of intensive care patients. Antimicrob Agents Chemother 2013:57:1488-95.

5. Harris PN, Tambyah PA, Paterson DL. $\beta$-Lactam and $\beta$-lactamase inhibitor combinations in the treatment of extended-spectrum $\beta$-lactamase producing Enterobacteriaceae: time for a reappraisal in the era of few antibiotic options? Lancet Infect Dis 2015;15:475-85.

6. Cantón R, Akóva M, Carmeli Y, et al. European Network on Carbapenemases. Rapid evolution and spread of carbapenemases among Enterobacteriaceae in Europe. Clin Microbiol Infect 2012;18:413-31.

7. Angus DC, van der Poll T. Severe sepsis and septic shock. $N$ Engl J Med 2013;369:840-51.

8. Esparcia A, Artero A, Eiros JM, et al. Influence of inadequate antimicrobial therapy on prognosis in elderly patients with severe urinary tract infections. Eur J Intern Med 2014;25:523-7.

9. Kumar A, Roberts D, Wood KE, et al. Duration of hypotension before initiation of effective antimicrobial therapy is the critical determinant of survival in human septic shock. Crit Care Med 2006;34:1589-96.

10. Parienti JJ, Lucet JC, Lefort A, et al. Empirical therapies among adults hospitalized for community-acquired upper urinary tract infections: a decision-tree analysis of mortality, costs, and resistance. Am J Infect Control 2015;43:e53-9

11. Peralta G, Lamelo $M$, Alvarez-García $P$, et al. Impact of empirical treatment in extended-spectrum beta-lactamase-producing Escherichia coli and Klebsiella spp. bacteremia. A multicentric cohort study. BMC Infect Dis 2012;12:245. 
12. Asakura $T$, Ikeda $M$, Nakamura $A$, et al. Efficacy of empirical therapy with non-carbapenems for urinary tract infections with extended-spectrum beta-lactamase-producing Enterobacteriaceae. Int J Infect Dis 2014;29:91-5.

13. Wie SH, Kim HW, Chang Ul. Effects of gentamicin monotherapy for the initial treatment of community-onset complicated non-obstructive acute pyelonephritis due to Enterobacteriaceae in elderly and non-elderly women. Clin Microbiol Infect 2014;20:1211-18.

14. Oteo J, Pérez-Vázquez M, Campos J. Extended-spectrum [beta]-lactamase producing Escherichia coli: changing epidemiology and clinical impact. Curr Opin Infect Dis 2010;23:320-6.

15. McMurdo MET, Gillespie ND. Urinary tract infection in old age: over-diagnosis and over-treated. Age Ageing 2000;29:297-8.
16. Woodford HJ, George J. Diagnosis and management of urinary tract infection in hospitalized older people. J Am Geriatr Soc 2009;57:107-14.

17. Colodner R. Extended-spectrum beta-lactamases: a challenge for clinical microbiologists and infection control specialists. Am J Infect Control 2005;33:104-7.

18. Brolund A. Overview of ESBL-producing Enterobacteriaceae from a Nordic perspective. Infect Ecol Epidemiol 2014;1:4.

19. Doi Y, Park YS, Rivera JI, et al. Community-associated extended-spectrum $\beta$-lactamase-producing Escherichia coli infection in the United States. Clin Infect Dis 2013:641-8.

20. Sundvall PD, Elm M, Gunnarsson R, et al. Antimicrobial resistance in urinary pathogens among Swedish nursing home residents remains low: a cross-sectional study comparing antimicrobial resistance from 2003 to 2012. BMC Geriatr. 2014;14:30. 